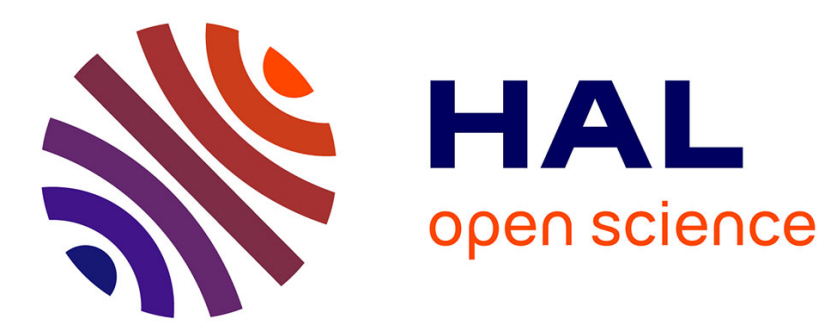

\title{
On the Stability of Polynomial Interpolation Using Hierarchical Sampling
}

\author{
Albert Cohen, Abdellah Chkifa
}

\section{To cite this version:}

Albert Cohen, Abdellah Chkifa. On the Stability of Polynomial Interpolation Using Hierarchical Sampling. Sampling Theory - A renaissance, pp.437-458, 2015, 10.1007/978-3-319-19749-4_12 . hal01353241

\section{HAL Id: hal-01353241 \\ https://hal.science/hal-01353241}

Submitted on 11 Aug 2016

HAL is a multi-disciplinary open access archive for the deposit and dissemination of scientific research documents, whether they are published or not. The documents may come from teaching and research institutions in France or abroad, or from public or private research centers.
L'archive ouverte pluridisciplinaire HAL, est destinée au dépôt et à la diffusion de documents scientifiques de niveau recherche, publiés ou non, émanant des établissements d'enseignement et de recherche français ou étrangers, des laboratoires publics ou privés. 


\title{
On the stability of polynomial interpolation using hierarchical sampling
}

\author{
Albert Cohen and Abdellah Chkifa
}

May 2, 2014

\begin{abstract}
Motivated by the development of non-intrusive methods for high dimensional parametric PDE's, we study the stability of a sparse high dimensional polynomial interpolation procedure introduced in [6]. A key aspect of this procedure is its hierarchical structure: the sampling set is progressively enriched together with the polynomial space. The evaluation points are selected from a grid obtained by tensorization of a univariate sequence. The Lebesgue constant that quantifies the stability of the resulting interpolation operator depends on the choice of this sequence. Here we study the $\Re$-Leja sequence, obtained by the projection of Leja sequences on the complex unit circle, with initial value 1 , onto $[-1,1]$. For this sequence, we prove cubic growth in the number of points for the Lebesgue constant of the multivariate interpolation operator, independently of the number of variable and of the shape of the polynomial space.
\end{abstract}

\section{Introduction}

This paper deals with a process of high dimensional approximation process, for which the sampling set is hierarchically enriched, in parallell with the polynomial space. Our main motivation for considering this process is the development of non-intrusive methods for high dimensional parametric PDE's.

Parametric PDE's are equations with the general form

$$
\mathcal{D}(u, y)=0
$$

where $\mathcal{D}$ is a differential operator and $y=\left(y_{1}, \ldots, y_{d}\right)$ is a parameter vector in a tensor product domain $X^{d}$. Up to a change of variable, typical choices for $X$ are the real interval $[-1,1]$ or the complex unit disk $\{|z| \leq 1\}$. The solution $u$ to such PDE's is therefore a function 
of $y$, which may be deterministic or stochastic depending on the context of application, in addition to the usual space and time variable.

Parametric PDE's raise significant computational challenges in the high dimensional context, that is when $d>>1$ or $d=+\infty$. Recent results such as in [9, 10, 7] have shown the effectiveness of approximating the map $y \mapsto u(y)$ to certain such PDE's by multivariate polynomials in the parametric variables $\left(y_{1}, \ldots, y_{d}\right)$. Here, the multivariate polynomial spaces are of the general form

$$
\mathbb{P}_{\Lambda}:=\operatorname{Span}\left\{y^{\nu}=y_{1}^{\nu_{1}} \ldots y_{d}^{\nu_{d}}: \nu=\left(\nu_{1}, \ldots, \nu_{d}\right) \in \Lambda\right\}
$$

where $\Lambda \in \mathbb{N}^{d}$ is an index set that is assumed to be downward closed (also called lower set), in the sense that

$$
\nu \in \Lambda \text { and } \mu_{i} \leq \nu_{i}, \quad i=1, \ldots, d \Rightarrow \mu \in \Lambda
$$

It was shown in [8, 7] that for relevant classes of parametric PDE's, certain sequences of downward closed index sets

$$
\Lambda_{1} \subset \Lambda_{2} \subset \ldots \subset \mathbb{N}^{d}
$$

with $\#\left(\Lambda_{k}\right)=k$ break the curse of dimensionality in the sense that the polynomial approximation error decays with $k$ at a rate $k^{-s}$ that does not deteriorates as $d$ gets large in the sense that it remains valid even when $d=\infty$.

One practical way to construct such polynomial approximations is by interpolation, based on the evaluation of $u$ at certain points $y^{i} \in X^{d}$. One attractive feature of such an approach is that it is non-intrusive and therefore can benefit from existing numerical codes for evaluating $y \mapsto u(y)$ pointwise. An important issue for computational simplicity and economy is that the sampling and interpolation procedure should be hierarchical: the solution $u$ is evaluated at only one new point in $X^{d}$ when $\Lambda_{k}$ is updated to $\Lambda_{k+1}$.

Such a procedure was recently proposed and analyzed in [6]. It is based on the data of a sequence $Z:=\left(z_{i}\right)_{i \geq 0}$ of pairwise distinct points in $X$, and the univariate interpolation operator $I_{k}$ onto $\mathbb{P}_{k}$ associated with the section $\left\{z_{0}, \cdots, z_{k}\right\}$. The corresponding multivariate interpolation operator $I_{\Lambda}$ onto $\mathbb{P}_{\Lambda}$ is constructed by a certain process of tensorization and sparsification based on the difference operators $\mathbb{D}_{k}:=I_{k}-I_{k-1}$, which is described in $\S 2$ of this paper. We also show that there is a simple relation between the algebraic growth of the Lebesgue constant $\mathbb{L}_{\Lambda}:=\left\|I_{\Lambda}\right\|_{L^{\infty} \rightarrow \mathbb{L}^{\infty}}$ in terms ot $\#(\Lambda)$, and that of its univariate counterpart $\mathbb{L}_{k}:=\left\|I_{k}\right\|_{L^{\infty} \rightarrow L^{\infty}}$ or of $\left\|\mathbb{D}_{k}\right\|_{L^{\infty} \rightarrow L^{\infty}}$ in terms of $k$.

This motivates the search for "good" univariate sequences $Z$ of points on $[-1,1]$ such that the, Lebesgue constant $\mathbb{L}_{k}$ or the norm of the difference operator $\mathbb{D}_{k}$, have moderate algebraic growth, controlled by $(1+k)^{\theta}$ for a small $\theta$. Note that is well known that the Lebesgue constant grows logarithmically with $k$ for certain choices of non-nested sets of 
points, such as Chebychev or Gauss-Lobatto points, however it is not clear that such a very slow growth is possible for nested sets corresponding to the sections of a sequence $Z$.

In this paper, we consider the so-called $\Re$-Leja sequence, obtained by the projection of Leja sequences on the complex unit circle, with initial value 1 , onto $[-1,1]$, and studied in [3, 4]. We recall in $\S 3$ some main properties of these sequences. We then obtain in $\S 4$ the bound $\mathbb{L}_{k} \leq 8 \sqrt{2}(1+k)^{2}$, which improves on the $\mathcal{O}\left(k^{3} \log k\right)$ bound in [3] and on the $\mathcal{O}\left(k^{2} \log k\right)$ bound in [4]. Then in $\S 5$, we establish the improved bound $\mathbb{D}_{k} \leq(1+k)^{2}$ for the difference operator, which could not be obtained directly from $\mathbb{D}_{k} \leq \mathbb{L}_{k}+\mathbb{L}_{k-1}$. A consequence of this last result is that using the $\Re$-Leja sequence, the resulting multivariate interpolation operator has Lebesgue constant with bound

$$
\mathbb{L}_{\Lambda} \leq(\# \Lambda)^{3},
$$

whatever the dimension $d$ and the shape of the finite lower set $\Lambda$.

\section{Sparse polynomial interpolation}

In this section, we recall the construction of the multivariate interpolation operator proposed in [6]. Given an infinite sequence $Z:=\left(z_{i}\right)_{i>0}$ of pairwise distinct points in $X$, we define $I_{k}$ the univariate interpolation operator onto $\mathbb{P}_{k}$ associated with the section $\left\{z_{0}, \cdots, z_{k}\right\}$. We may express $I_{k}$ as the telescoping sum

$$
I_{k}=\sum_{l=0}^{k} \Delta_{l}, \quad \Delta_{0}=I_{0} \text { and } \Delta_{k}:=I_{k}-I_{k-1},
$$

which corresponds to the Newton form, with

$$
\Delta_{k} f=\left(f\left(z_{k}\right)-I_{k-1} f\left(z_{k}\right)\right) h_{k}, \quad h_{0}(z)=1, \quad h_{k}(z)=\prod_{j=0}^{k-1} \frac{z-z_{j}}{z_{k}-z_{j}},
$$

with the convention that $I_{-1}=0$. Now, for an arbitrary lower set $\Lambda \subset \mathbb{N}^{d}$, we introduce the grid of points

$$
\Gamma_{\Lambda}:=\left\{z_{\nu}: \nu \in \Lambda\right\} \quad \text { where } \quad z_{\nu}:=\left(z_{\nu_{j}}\right)_{j=1, \ldots, d} \in X^{d}
$$

We also introduce the operator

$$
I_{\Lambda}:=\sum_{\nu \in \Lambda} \Delta_{\nu}, \quad \Delta_{\nu}:=\otimes_{j=1, \ldots, d} \Delta_{\nu_{j}}
$$

We observe that this coincides with 2.1 for the univariate case $d=1$ when $\Lambda=\{0,1, \ldots, k\}$. We also observe that when $\Lambda$ is a rectangular block, that is,

$$
\Lambda=\mathcal{B}_{\mu}:=\{\nu: \nu \leq \mu\}
$$


for some $\mu$, then

$$
I_{\Lambda}=\otimes_{j=1, \ldots, d}\left(\sum_{\nu_{j}=1}^{\mu_{j}} \Delta_{\nu_{j}}\right)=\otimes_{j=1, \ldots, d} I_{\mu_{j}},
$$

is the interpolation operator for the tensor product polynomial space $\mathbb{P}_{\Lambda}:=\otimes_{j=1, \ldots, d} \mathbb{P}_{\mu_{j}}$ for the tensor product grid $\Gamma_{\Lambda}=\otimes_{j=1, \ldots, d}\left\{z_{0}, \ldots, z_{\mu_{j}}\right\}$.

The following result is given in [6] but its first appearance dates back from [14] in the bi-dimensional case. It shows that this observation generalizes to any downward closed set.

Theorem 2.1 The grid $\Gamma_{\Lambda}$ is unisolvant for the polynomial space $\mathbb{P}_{\Lambda}$ and that the interpolation operator is given by $I_{\Lambda}$.

Proof: Since $\#(\Lambda)=\operatorname{dim}\left(\mathbb{P}_{\Lambda}\right)$ and the image of $I_{\Lambda}$ is obviously contained in $\mathbb{P}_{\Lambda}$, it suffices to show that $I_{\Lambda}$ is the interpolation operator, that is, $I_{\Lambda} f\left(z_{\mu}\right)=f\left(z_{\mu}\right)$ for all $\nu \in \Lambda$. This is shown by splitting $I_{\Lambda} f$ into

$$
I_{\Lambda} f=I_{\mathcal{B}_{\mu}} f+\left(I_{\Lambda}-I_{\mathcal{B}_{\mu}}\right) f,
$$

where $\mathcal{B}_{\mu}$ is the rectangular block in (2.5). For the first, we have already observed that $I_{\mathcal{B}_{\mu}} f\left(z_{\mu}\right)=f\left(z_{\mu}\right)$. The second part in the above splitting is a sum of terms $\Delta_{\nu} f$ where $\nu$ is such that $\nu_{j}>\mu_{j}$ for at least one value of $j$. For this value we have $\Delta_{\nu_{j}} f\left(z_{\mu_{j}}\right)=0$, which implies that $\Delta_{\nu} f\left(z_{\mu}\right)=0$. Therefore $\left(I_{\Lambda}-I_{\mathcal{B}_{\mu}}\right) f\left(z_{\mu}\right)=0$ which concludes the proof.

One main interest of the above construction is that it is hierarchical in the sense that the enrichment of $\Lambda$ by a new index $\mu$ corresponds to adding one sampling point $z_{\mu}$ to the grid $\Gamma_{\Lambda}$. In a similar way to the univariate case, the hierarchical computation of the interpolant is possible, based on the formula

$$
\Delta_{\nu} f=\left(f\left(z_{\nu}\right)-I_{\Lambda} f\left(z_{\nu}\right)\right) H_{\nu}, \quad H_{\nu}(z)=\prod_{j=1}^{d} h_{\nu_{j}}\left(z_{j}\right),
$$

which holds whenever $\Lambda$ is any lower set such that any $\nu \notin \Lambda$ and $\Lambda \cup\{\nu\}$ is also a lower set. This hierarchical form allows us to develop adaptive interpolation algorithms: given a certain set $\Lambda_{n}$ of cardinality $n$, one picks a new index $\nu^{n+1}$ which maximizes the contribution $\Delta_{\nu} f$ in some norm of interest (typically $L^{p}$ for $p=1,2$ or $\infty$ ) among those $\nu \notin \Lambda_{n}$ such that $\Lambda_{n} \cup\{\nu\}$ is a lower set. The numerical behaviour of such adaptive algorithms is studied in [6].

The stability of the operators $I_{\Lambda}$ is critical for numerical applications such as the nonintrusive treatment of parametric PDE's. It is measured by the Lebesgue constant

$$
\mathbb{L}_{\Lambda}:=\max _{f \in C\left(X^{d}\right)-\{0\}} \frac{\left\|I_{\Lambda} f\right\|_{L^{\infty}\left(X^{d}\right)}}{\|f\|_{L^{\infty}\left(X^{d}\right)}} .
$$


In particular, we have the classical estimate

$$
\left\|f-I_{\Lambda} f\right\|_{L^{\infty}\left(X^{d}\right)} \leq\left(1+\mathbb{L}_{\Lambda}\right) \inf _{g \in \mathbb{P}_{\Lambda}}\|f-g\|_{L^{\infty}\left(X^{d}\right)} .
$$

This constant depends on the sequence $Z$, in particular through the Lebesgue constant of the univariate interpolation operators

$$
\mathbb{L}_{k}:=\max _{f \in C(X)-\{0\}} \frac{\left\|I_{k} f\right\|_{L^{\infty}(X)}}{\|f\|_{L^{\infty}(X)}} .
$$

We recall that

$$
\mathbb{L}_{k}:=\max _{t \in X} \lambda_{k}(t)
$$

where $\lambda_{k}$ is the Lagrange function for the section $\left\{z_{0}, \ldots, z_{k}\right\}$ defined by

$$
\lambda_{k}(t):=\sum_{i=1}^{k}\left|l_{i, k}(t)\right|,
$$

with

$$
l_{i, k}(t):=\prod_{\substack{j=1, \ldots, k, j \neq i}} \frac{t-z_{j}}{z_{i}-z_{j}}
$$

for $j=0, \ldots, k$ are the Lagrange polynomials associated with $\left\{z_{0}, \ldots, z_{k}\right\}$.

It is shown in [6] that algebraic growth of $\mathbb{L}_{k}$ yields algebraic growth of the Lebesgue constant $\mathbb{L}_{\Lambda}$. More precisely, given any $\theta \geq 1$

$$
\mathbb{L}_{k} \leq(1+k)^{\theta}, \quad \text { for any } \quad k \geq 1 \quad \Longrightarrow \quad \mathbb{L}_{\Lambda} \leq(\# \Lambda)^{\theta+1} .
$$

Surprisingly, the previous implication is valid whatever the dimension $d$ and the shape of the finite lower set $\Lambda$.

A more straightforward computation shows that we also have

$$
\mathbb{D}_{k} \leq(1+k)^{\theta}, \quad \text { for any } \quad k \geq 1 \quad \Longrightarrow \quad \mathbb{L}_{\Lambda} \leq(\# \Lambda)^{\theta+1},
$$

where

$$
\mathbb{D}_{k}:=\max _{f \in C(X)-\{0\}} \frac{\left\|\Delta_{k} f\right\|_{L^{\infty}(X)}}{\|f\|_{L^{\infty}(X)}} .
$$

Indeed, by triangle inequality, we find that

$$
\mathbb{L}_{\Lambda} \leq \sum_{\nu \in \Lambda} \prod_{j=1}^{d} \mathbb{D}_{\nu_{j}} \leq \sum_{\nu \in \Lambda} \prod_{j=1}^{d}\left(1+\nu_{j}\right)^{\theta}=\sum_{\nu \in \Lambda}\left(\#\left(\mathcal{B}_{\nu}\right)\right)^{\theta} \leq \sum_{\nu \in \Lambda}(\# \Lambda)^{\theta}=(\# \Lambda)^{\theta+1},
$$

where in the forth inequality, we have used the fact that $\mathcal{B}_{\nu} \subset \Lambda$ for any $\nu \in \Lambda$ because $\Lambda$ is downward closed. 
The construction of sequences with algebraic growth of the Lebesgue constant is then essential. In all the following, without loss of generality, we consider the interval $X=[-1,1]$, for which the classical choices of Chebyshev and Gauss-Lobatto points gives univariate Lebesgue constants that grow polynomially with $k$. However, these choices are of no use for our purposes since they do not correspond to the sections of a single sequence $Z$.

A possible alternative is provided by the so-called Leja sequences $A:=\left(a_{j}\right)_{j \geq 0}$ constructed according to: $a_{0} \in[-1,1]$ arbitrary and $a_{k}$ satisfying

$$
\left|a_{k}-a_{0}\right| \ldots\left|a_{k}-a_{k-1}\right|=\max _{t \in[-1,1]}\left|t-a_{0}\right| \ldots\left|t-a_{k-1}\right|
$$

Numerical evidence shows that such sequences have moderate growth of the Lebesgue constant, the bound $\mathbb{L}_{k} \leq k$ seems valid, see [4]. However, no rigorous proof supports this evidence. It is only known that the growth of the Lebesgue constants is sub-exponential, i.e. $\left(\mathbb{L}_{k}\right)^{\frac{1}{k}} \rightarrow_{k \rightarrow \infty} 0$, see [15]. In the rest of this paper, we provide estimates on the growth of Lebesgue constants for slightly different sequences, namely Leja points for the complex unit disk and their projections on the interval $[-1,1]$.

\section{Leja sequences and their projections}

\subsection{Leja sequence on the unit circle}

Recently, Calvi and Phung [2, 3] have shown that the Lebesgue constants of Leja sequences on $\mathcal{U}$ the unit disk and theirs real projection on $[-1,1]$, the so-called $\Re$-Leja sequences, are moderate and have growth asymptotically bounded in $\mathcal{O}(k \log k)$ and $\mathcal{O}\left(k^{3} \log k\right)$ respectively. In addition, unlike Leja sequences on $[-1,1]$, theses sequences are easy to construct and have explicit formulas. In [4], their bounds were improved to $2 k$ and $5 k^{2} \log k$, respectively. In this paper, we improve further these bounds and give direct bounds for the norms $\mathbb{D}_{k}$ of the difference operators, which are useful in view of the discussion in the previous section. Our techniques of proof share several common points with those developed in [2, 3, 4, yet it is shorter and exploit to a considerable extent the properties of Leja sequences on the unit disk.

We introduce the notations $\mathcal{U}$ and $\partial \mathcal{U}$ for the closed complex unit disk and the complex unit circle respectively and the notation $\mathcal{U}_{N}$ for the set of $N$-root of unity. Given an infinite sequence $A:=\left(a_{j}\right)_{j \geq 0}$, we define $A_{k}:=\left(a_{0}, \cdots, a_{k-1}\right)$ and $A_{l, m}=\left(a_{l}, \cdots, a_{m}\right)$ for $l \leq m$. Given two finite sequence $S_{1}$ and $S_{2}$, we denote by $S_{1} \wedge S_{2}$ the concatenation of $S_{1}$ and $S_{2}$. For any section $S=\left(s_{0}, \cdots, s_{l}\right)$ of complex number, we introduce the notations

$$
\rho S:=\left(\rho s_{0}, \cdots, \rho s_{l}\right), \rho \in \mathbb{C}, \quad \Re(S):=\left(\Re\left(s_{0}\right), \cdots, \Re\left(s_{l}\right)\right), \quad \bar{S}:=\left(\overline{s_{0}}, \cdots, \overline{s_{l}}\right) .
$$


Throughout this paper, to any finite set $S$ of numbers, we associate the polynomial

$$
w_{S}(x):=\prod_{s \in S}(x-s)
$$

Any integer $k \geq 1$ can be uniquely expanded according to

$$
k=2^{p_{0}}+\ldots+2^{p_{s_{k}-1}}, \quad p_{0}<\ldots<p_{s_{k}-1},
$$

where $s_{k}$ is the number of ones in the binary representation of $k$ and the $p_{j}$ 's are integers. We emphasize the dependance of $p_{0}$ in $k$ when needed by sometimes writing $p_{0}(k)$. We denote by $\sigma_{1}(k)$ and $\sigma_{0}(k)$ respectively, the number of ones and zeros in the binary expression of $k$. For $k=2^{n}, \ldots, 2^{n+1}-1$, one has

$$
\sigma_{1}(k)=s_{k} \leq n, \quad \sigma_{0}(k)=n+1-\sigma_{1}(k)
$$

We recall also that for any $n \geq 1$ and any $0<l<2^{n}$, one has

$$
s_{l}+s_{2^{n}-l}=n+1-p_{0}(l)
$$

The proof is simple and can be found in [4].

Leja sequences $E=\left(e_{j}\right)_{j \geq 0}$ on $\mathcal{U}$ considered in [2, 4] have all theirs initial value $e_{0} \in \partial \mathcal{U}$ the unit circle. In view of the definition (3.6), the maximum principle implies $e_{j} \in \partial \mathcal{U}$ for any $j \geq 1$. The sequence considered in [2] are actually Leja sequence on the unit circle.

A Leja sequence on the unit circle $E=\left(e_{j}\right)_{j \geq 0}$ is defined inductively by: pick $e_{0} \in \partial \mathcal{U}$ arbitrary and for $k \geq 1$

$$
e_{k}=\operatorname{argmax}_{z \in \partial \mathcal{U}}\left|z-e_{k-1}\right| \ldots\left|z-e_{0}\right|
$$

The previous argmax problem might admit many solutions and $e_{k}$ is one of them. We call a $k$-Leja section every finite sequence $\left(a_{0}, \ldots, a_{k-1}\right)$ obtained by the same recursive procedure. In particular, with $E$ is a sequence as above, then the section $E_{k}=\left(e_{0}, \ldots, e_{k-1}\right)$ is $k$-Leja section.

In contrast to the interval $[-1,1]$ where even the first points of a Leja sequence can not be computed explicitly, Leja sequences on $\partial \mathcal{U}$ are much easier to compute. For instance, suppose that $e_{0}=1$, then we can immediately check that $e_{1}=-1$ and $e_{2}= \pm i$. Assume that $e_{2}=i$ then $e_{3}$ maximises $\left|z^{2}-1\right||z-i|$, so that $e_{3}=-i$ because $-i$ maximizes jointly $\left|z^{2}-1\right|$ and $|z-i|$. Then $e_{4}$ must maximize $\left|z^{4}-1\right|$, etc... We observe that a "binary" patten on the distribution of $E$ begin to appear.

Since the element of $\partial \mathcal{U}$ have all the same modulus 1 , then an arbitrary Leja sequence $E=\left(e_{0}, \ldots\right)$ on $\partial \mathcal{U}$ is merely the rotation by $e_{0}$ of a Leja sequence with initial value 1 . The latter are completely determined according to the following theorem, see [2, 4]. 
Theorem 3.1 Let $n \geq 0,2^{n}<k \leq 2^{n+1}$ and $l=k-2^{n}$. The finite sequence $E_{k}=$ $\left(e_{0}, \ldots, e_{k-1}\right)$ is a $k$-Leja section if and only if $E_{2^{n}}=\left(e_{0}, \ldots, e_{2^{n}-1}\right)$ and $U_{l}=\left(e_{2^{n}}, \ldots, e_{k-1}\right)$ are respectively $2^{n}$-Leja and $l$-Leja sections and $e_{2^{n}}$ is any $2^{n}$-root of -1 .

The most natural construction of a Leja sequence in $\partial U$ consists then in defining $E:=$ $\left(e_{j}\right)_{j \geq 0}$ inductively by

$$
E_{1}:=\left(e_{0}=1\right) \quad \text { and } \quad E_{2^{n+1}}:=E_{2^{n}} \wedge e^{\frac{i \pi}{2^{n}}} E_{2^{n}}, \quad n \geq 0 .
$$

This "uniform" construction of the sequence $E$ yields an interesting distribution of its elements. Indeed, by an immediate induction, see [1], it can be shown that the elements $e_{k}$ are given by

$$
e_{k}=\exp \left(i \pi \sum_{l=0}^{n} a_{j} 2^{-j}\right) \quad \text { for } \quad k=\sum_{j=0}^{s} a_{j} 2^{j}, \quad a_{j} \in\{0,1\} .
$$

The construction yields then a low-discrepancy sequence on $\partial \mathcal{U}$ based on the bit-reversal Van der Corput enumeration. This sequence was known to be a Leja sequence over $\partial \mathcal{U}$ in many earlier works.

As stated above, Theorem 3.1 characterizes completely Leja sequence on the unit circle. It has many implications that turn out to be very useful in the analysis of the growth of Lebesgue constant studied. We have

Theorem 3.2 Let $E$ be a Leja sequence on $\partial \mathcal{U}$ starting at 1 . The following holds:

- For any $n \geq 0, E_{2^{n}}=\mathcal{U}_{2^{n}}$ in the set sense.

- For any $k \geq 1,\left|w_{E_{k}}\left(e_{k}\right)\right|=\sup _{z \in \partial \mathcal{U}}\left|w_{E_{k}}(z)\right|=2^{\sigma_{1}(k)}$.

- For any $n \geq 0, E_{2^{n}, 2^{n+1}}:=\left(e_{2^{n}}, \cdots, e_{2^{n+1}-1}\right)$ is a $2^{n}$-Leja section.

- For any $n \geq 0, \mathcal{B}\left(E_{2^{n}}\right):=\left(e_{2^{n}-1}, \cdots, e_{1}, e_{0}\right)$ is a $2^{n}$-Leja section.

- The sequence $E^{2}:=\left(e_{2 j}^{2}\right)_{j \geq 0}$ is a Leja sequence.

The proof of the properties can be found in [2, 4, 5].

Using the implications of the Leja definition (3.6) on the growth of the Lebesgue constants $\mathbb{L}_{E_{k}}$ of the sections $E_{k}$ and the previous structural properties of Leja sequences on the unit circle, it was proved in [4] that for any Leja sequence $E$ on $\partial \mathcal{U}$, we have

$$
\lambda_{E_{k}}\left(e_{k}\right) \leq k \text { and } \mathbb{L}_{E_{k}} \leq 2 k, \quad k \geq 1,
$$

where $\lambda_{E_{k}}$ is the Lagrange function associated with the section $E_{k}$ which is defined in a similar manner as in 2.13. 
For further use, let us note that given $E$ a Leja section starting at $\rho \in \partial U, n \geq 1$ and $k$ such that $1 \leq k \leq 2^{n}$, one has for any $z, \xi \in \partial \mathcal{U}$ with $\xi \notin E_{k}$

$$
\frac{\left|w_{E_{k}}(z)\right|}{\left|w_{E_{k}}(\xi)\right|}=\frac{\left|w_{E_{k}}(z)\right|\left|w_{\mathcal{B}\left(E_{k, 2^{n}}\right)}(\xi)\right|}{\left|w_{E_{2^{n}}}(\xi)\right|} \leq \frac{2^{\sigma_{1}(k)} 2^{\sigma_{1}\left(2^{n}-k\right)}}{\left|\xi^{2^{n}}-\rho^{2^{n}}\right|}=\frac{2^{n+1-p_{0}(k)}}{\left|\xi^{2^{n}}-e_{0}^{2^{n}}\right|} .
$$

We have used that $E_{k} \cup \mathcal{B}\left(E_{k, 2^{n}}\right)=E_{k} \cup E_{k, 2^{n}-1}=E_{2^{n}}=\rho \mathcal{U}_{2^{n}}$ in the set sense, that $\mathcal{B}\left(E_{k, 2^{n}}\right)$ is a $\left\{2^{n}-k\right\}$-Leja section according to the forth properties above, and the easily checked identity $\sigma_{1}(k)+\sigma_{1}\left(2^{n}-k\right)=n+1-p_{0}(k)$ for any $0 \leq k \leq 2^{n}$.

\section{$3.2 \quad \Re$-Leja sequences on $[-1,1]$}

We consider a Leja sequence $E=\left(e_{j}\right)_{j \geq 0}$ on the unit circle with $e_{0}=1$ and project it onto the real interval $[-1,1]$ and denote by $R=\left(r_{j}\right)_{j \geq 0}$ the sequence obtained. Since $E=(1,-1, \pm i, \cdots)$, one should make sure that no point is repeated on $R$ simply by not projecting a point $e_{j}$ such that $e_{j}=\bar{e}_{i}$ for some $i<j$. Such sequences $R$ were named $\Re$-Leja sequence in [3]. The projection rule that prevent the repetition is well understood. Indeed, it was in proved in [3, Theorem 2.4] that

Lemma 3.3 Let $E$ be a Leja sequence on $\partial \mathcal{U}$ with $e_{0}=1$ and $R$ the associated $\Re$-Leja sequence. Then

$$
R=\Re(Z), \quad \text { with } \quad Z:=(1,-1) \wedge \bigwedge_{j=1}^{\infty} E_{2^{j}, 2^{j}+2^{j-1}}
$$

The previous theorem says essentially that the section $E_{2^{n}, 2^{n+1}}$ considered as a set is the union of its first half $E_{2^{n}, 2^{n}+2^{n-1}}$ and its conjugates. $\overline{E_{2^{n}, 2^{n}+2^{n-1}}}$.

A straightforward cardinality argument shows that in addition to $r_{0}=1, r_{1}=-1$, we have for any $n \geq 0$ and any $k$ with $2^{n} \leq k-1<2^{n+1}$,

$$
Z_{k}=(1,-1) \wedge \bigwedge_{j=1}^{n} E_{2^{j}, 2^{j}+2^{j-1}} \wedge E_{2^{n+1}, 2^{n}+k-1} \quad \text { and } \quad r_{k}=\Re\left(e_{2^{n}+k-1}\right) .
$$

The particular structure of the Leja sequences $E$ yields useful properties for $\Re$-Leja sequences. First, in view of the first property in Theorem 3.2, we have

$$
R_{2^{n}+1}=\left\{\cos \left(\frac{j \pi}{2^{n}}\right): j=0, \ldots, 2^{n}\right\}, \quad n \geq 0
$$

in the set sense. Therefore $R_{2^{n}+1}$ coincides as a set with the Gauss-Lobatto abscissas. We have also the following result. 
Lemma 3.4 Let $R:=\left(r_{j}\right)_{j \geq 0}$ be a $\Re$-Leja sequence. The sequence

$$
R^{2}:=\left(2 r_{2 j}^{2}-1\right)_{j \geq 0}
$$

is also an $\Re$-Leja sequence.

Proof: We consider $E=\left(e_{j}\right)_{j \geq 0}$ to be a Leja sequence associated with $R$ and recall that by Theorem 3.2, the sequence $E^{2}=\left(e_{2 j}^{2}\right)_{j \geq 0}$ is also Leja sequence starting at 1 since $e_{0}=1$. the sequence $R^{2}$ can be obtained by projection of $E^{2}$ onto [-1,1]. Indeed, the first two elements of $R^{2}$ are 1 and -1 because $r_{0}=1, r_{2}=0$, so that we only need to show that 3.12 holds with $R^{2}$ and $E^{2}$. For $n \geq 0$ and $2^{n} \leq k-1<2^{n+1}$, one has $2^{n+1} \leq(2 k-1)-1<2^{n+2}$ so that by 3.12 ,

$$
r_{2 k-1}=\Re\left(e_{2^{n+1}+2 k-1-1}\right)=\Re\left(e_{2\left(2^{n}+k-1\right)}\right) .
$$

Since $2 k \geq 4$, then $r_{2 k}=-r_{2 k-1}$, hence

$$
2 r_{2 k}^{2}-1=2 r_{2 k-1}^{2}-1=\Re\left(e_{2\left(2^{n}+k-1\right)}^{2}\right),
$$

where we have used $\Re\left(z^{2}\right)=2 \Re(z)^{2}-1$ for $z \in \partial \mathcal{U}$. The proof is then complete.

The previous lemma has certain implications on the polynomials $w_{R_{k}}$ associated with the sections $R_{k}$ which are very essential on the study of the growth of the norm of the difference operator discussed in section $\S 5$. In order to lighten our notation, we find it convenient to work with normalized versions of the polynomials $w_{R_{k}}$ that we define by

$$
W_{R_{k}}(x):=2^{k} w_{R_{k}}(x), \quad x \in[-1,1] .
$$

We are interested in the relation between these polynomials for sections of the sequences $R$ and $R^{2}$. First, since all $\Re$-Leja sequences has initial elements 1 and -1 , then it is immediate that

$$
W_{R_{1}^{2}}\left(2 x^{2}-1\right)=W_{R_{2}}(x) \quad x \in[-1,1] .
$$

For higher value of $k$, we have the following

Lemma 3.5 Let $R$ be an $\Re$-Leja sequence and $S:=R^{2}$. For any $k \geq 2$

$$
W_{S_{k}}\left(2 x^{2}-1\right)=2 x W_{R_{2 k-1}}(x), \quad x \in[-1,1]
$$

Consequently $W_{S_{k}}^{\prime}(-1)=W_{R_{2 k-1}}^{\prime}(0), W_{S_{k}}^{\prime}(1)=\frac{1}{2} W_{R_{2 k-1}}^{\prime}(1)=\frac{1}{2} W_{R_{2 k-1}}^{\prime}(-1)$ and

$$
W_{S_{k}}^{\prime}\left(s_{j}\right)=\frac{1}{2} W_{R_{2 k-1}}^{\prime}\left(r_{2 j}\right)=\frac{1}{2} W_{R_{2 k-1}}^{\prime}\left(r_{2 j-1}\right), \quad j=2, \ldots, k-1
$$


Proof: The verification of 3.17 for $k=2$ is immediate. Now, from the definition of $R^{2}$, we have for $k \geq 3$

$$
w_{S_{k}}\left(2 x^{2}-1\right)=\prod_{j=0}^{k-1}\left(2 x^{2}-1-\left(2 r_{2 j}^{2}-1\right)\right)=2^{k} \prod_{j=0}^{k-1}\left(x+r_{2 j}\right)\left(x-r_{2 j}\right) .
$$

Since $r_{0}=1, r_{1}=-1, r_{2}=0$ and $r_{2 j}=-r_{2 j-1}$ for any $j \geq 2$, then

$$
w_{S_{k}}\left(2 x^{2}-1\right)=2^{k}(x+1)(x-1) x^{2} \prod_{j=2}^{k-1}\left(x-r_{2 j-1}\right)\left(x-r_{2 j}\right)=2^{k} x w_{R_{2 k-1}}(x),
$$

which implies (3.17) after multiplication by $2^{k}$. The derivation with respect to $x$ gives

$$
4 x W_{S_{k}}^{\prime}\left(2 x^{2}-1\right)=2\left(x W_{R_{2 k-1}}^{\prime}(x)+W_{R_{2 k-1}}(x)\right) .
$$

Since $W_{R_{2 k-1}}(0)=0$, then the first result on derivatives is obtained when dividing by $x$ and letting $x \rightarrow 0$. The second result is obtained by the substitution of $x$ by 1 or -1 . As for (3.18, we substitute $x$ by $r_{2 j}$ and $r_{2 j-1}=-r_{2 j}$ for $j=2 \ldots, k-1$.

The previous Lemma has also implications on the growth of $W_{R_{k}}\left(r_{k}\right)$ that we use in $\S 4$.

Lemma 3.6 Let $R$ be a $\Re$-Leja sequence and denote $S:=R^{2}$. For any $N \geq 1$, we have

$$
2 r_{k} W_{R_{k}}\left(r_{k}\right)=W_{S_{N+1}}\left(s_{N+1}\right), \quad k=2 N+1,
$$

and

$$
W_{R_{k}}\left(r_{k}\right)=2 W_{S_{N}}\left(s_{N}\right), \quad k=2 N .
$$

Proof: The first equality follows from formula (3.17) applied with $x=r_{k}$ since $k=2(N+$ 1) -1 and $2 r_{k}^{2}-1=2 r_{2(N+1)}^{2}-1=s_{N+1}$. The second equality can be checked easily for $N=1$. For $N \geq 2$, using the fact $r_{k}=-r_{2 N-1}$ and $s_{N}=2 r_{k}^{2}-1$, formula 3.17 implies

$$
W_{R_{k}}\left(r_{k}\right)=2\left(r_{k}-r_{2 N-1}\right) W_{R_{2 N-1}}\left(r_{k}\right)=4 r_{k} W_{R_{2 N-1}}\left(r_{k}\right)=2 W_{S_{N}}\left(s_{N}\right) .
$$

\section{Growth of Lebesgue constant of $\Re$-Leja sections}

As stated above in (3.13), for any $\Re$-Leja sequence $R$, the sections $R_{2^{n}+1}$ coincide in the set sense with the Gauss-Lobatto abscissas. This type of abscissas are known to have Lebesgue constant with logarithmic growth $\mathbb{L}_{R_{2^{n}+1}} \sim \frac{2}{\pi} \log \left(2^{n}+1\right)$. More precisely, we have the bound

$$
\mathbb{L}_{R_{2^{n}+1}} \leq 1+\frac{2}{\pi} \log \left(2^{n}\right)
$$


See [12, Formulas 5 and 13]. In [4], using the previous bound and classical trigonometric arguments as the one used in the bounding of Lebesgue constant of Tchybeshev abscissas, e.g. [13], it is established that for any $n \geq 0$ and any $k \geq 2^{n}+1$

$$
\mathbb{L}_{R_{k}} \leq 4^{n-p_{0}\left(k^{\prime}\right)}\left(5+\frac{8}{\pi} \log 2^{n}\right)
$$

where $k^{\prime}=k-\left(2^{n}+1\right)$. Although the effect of the binary pattern on the distribution of the Leja sequence $E$ on $\partial \mathcal{U}$ is somehow reflected by the term $2^{n-p_{0}\left(k^{\prime}\right)}$, we observe that if $k$ is an even number, we only have the bound $\mathbb{L}_{R_{k}} \leq \frac{8}{\pi} k^{2} \log k$.

Through a novel analysis, we propose to relate the analysis of the Lebesgue constants $\mathbb{L}_{R_{k}}$ to the analysis of the Lebesgue constants $\mathbb{L}_{E_{k}}$ where $E$ is any Leja sequence associated with $R$, then benefit from the machinery developed for the complex setting in [4].

The sections $R_{k}$ of length $k=2^{n}+1$ for $n \geq 1$ have already been treated, see (4.1). Therefore, we only discuss the cases of $k$ such that $2^{n}+1<k<2^{n+1}+1$. In view of (3.12), for such values, we have $R_{k}=\Re\left(Z_{k}\right), Z_{k}$ being the section obtained by the elimination procedure from $E_{2^{n}+k-1}$ which is the shortest section of $E$ that yields $R_{k}$ we projected onto $[-1,1]$. We have the following result

Theorem 4.1 Let $n \geq 0$ and $k \geq 3$ such that $2^{n}+1<k<2^{n+1}+1$. One has

$$
\mathbb{L}_{R_{k}} \leq 2 \sqrt{2}\left(2^{n-p_{0}\left(k^{\prime}\right)} \mathbb{L}_{E_{k+2^{n}-1}}\right) \quad \text { where } \quad k^{\prime}=k-\left(2^{n}+1\right) .
$$

In view of (3.9), the previous theorem implies in particular

$$
\mathbb{L}_{R_{k}} \leq 2 \sqrt{2}\left(2^{n} \times 2\left(k+2^{n}-1\right)\right) \leq 8 \sqrt{2} k^{2}
$$

In order to prove the theorem, we must bound the Lebesgue function associated with the real section $R_{k}$ using the Lebesgue function or constant associated with the complex section $E_{k+2^{n}-1}$. To this end, we propose to bound the Lagrange polynomials associated with $R_{k}$ using those associated with $E_{k+2^{n}-1}$.

For notational simplicity, we introduce

$$
G_{k}=E_{k+2^{n}-1}, \quad 2^{n}+1<k<2^{n+1}+1,
$$

where $G_{k}$ is a set. The following lemma describe to some extent how $G_{k}$ can be obtained from $R_{k}$.

Lemma 4.2 Let $E$ be a Leja sequence with $e_{0}=1, R$ the associated $\Re$-Leja sequence, and $Z=\left(z_{j}\right)_{j \geq 0}$ the sequence in Lemma 3.3. For any $n \geq 0$ and any $k$ with $2^{n}+1<k<2^{n+1}+1$, we have

$$
G_{k}=\left\{z_{0}, z_{1}\right\} \cup\left\{z_{2}, \overline{z_{2}}, \cdots, z_{2^{n}}, \overline{z_{2^{n}}}\right\} \cup F_{k} \quad F_{k}:=Z_{2^{n}+1, k-1}=\left\{z_{2^{n}+1}, \cdots, z_{k-1}\right\} .
$$


Proof: We have that

$$
G_{k}=E_{2^{n+1}} \wedge E_{2^{n+1}, 2^{n}+k-1}=E_{2^{n+1}} \wedge Z_{2^{n}+1, k-1} .
$$

Therefore, we only need to show that $E_{2^{n+1}}=\left\{z_{0}, z_{1}, z_{2}, \overline{z_{2}}, \cdots, z_{2^{n}}, \overline{z_{2^{n}}}\right\}$ in the set sense. Since $E_{2^{n+1}}$ coincides with the set of $2^{n+1}$-root of unity, then $E_{2^{n}+1}$ is the union of $\{1,-1\}$ and $\left\{z_{2}, \ldots, z_{2^{n}}\right\}$ and theirs conjugates, which finishes the proof.

The previous Lemma allows us to relate the polynomials $W_{R_{k}}$ defined in 3.15 and $w_{G_{k}}$, and also their derivatives.

Lemma 4.3 Let $n, k, F_{k}$ and $G_{k}$ as in the previous lemma. For any $z \in \partial \mathcal{U}$ and $x=\Re(z)$

$$
\left|W_{R_{k}}(x)\right|=\left|z^{2}-1\right|\left|w_{G_{k}}(z)\right|\left|w_{\overline{F_{k}}}(z)\right|=\left|\bar{z}^{2}-1\right|\left|w_{G_{k}}(\bar{z})\right|\left|w_{\overline{F_{k}}}(\bar{z})\right| .
$$

Consequently, for any $j=0, \cdots, k-1$

$$
\left|W_{R_{k}}^{\prime}\left(r_{j}\right)\right|=2 \alpha_{j}\left|w_{G_{k}}^{\prime}\left(z_{j}\right)\right|\left|w_{\overline{F_{k}}}\left(z_{j}\right)\right|,
$$

where $\alpha_{j}=1$ for every $j$ except for $j=0$ and $j=1$, it is equal to 2 .

Proof: Given $z, z^{\prime} \in \partial \mathcal{U}$ and $x=\frac{1}{2}(z+\bar{z})$ and $x^{\prime}=\frac{1}{2}\left(z^{\prime}+\bar{z}^{\prime}\right)$, one easily checks that

$$
2\left|x-x^{\prime}\right|=\left|z-z^{\prime}\right|\left|z-\overline{z^{\prime}}\right| .
$$

Since $r_{j}=\Re\left(z_{j}\right)$ and $z_{j} \in \partial \mathcal{U}$ for any $j \geq 0$, then

$$
\left|W_{R_{k}}(x)\right|=\prod_{j=0}^{k-1} 2\left|x-r_{j}\right|=\prod_{j=0}^{k-1}\left|z-z_{j}\right| \prod_{j=0}^{k-1}\left|z-\overline{z_{j}}\right| .
$$

In view of (4.6), taking into account that $z_{0}=1$ and $z_{1}=-1$ are repeated twice in the previous product, the first part in (4.7) follows. The second part is immediate since $z$ and $\bar{z}$ play symmetric roles. This result combined with the identity 4.9 ), shows that for every $j=1, \cdots, k-1$

$$
\left|W_{R_{k}}^{\prime}\left(r_{j}\right)\right|=\lim _{x \rightarrow r_{j}} \frac{\left|W_{R_{k}}(x)\right|}{\left|x-r_{j}\right|}=\lim _{z \rightarrow z_{j}} \frac{\left|z^{2}-1\right|\left|w_{G_{k}}(z)\right|\left|w_{\overline{F_{k}}}(z)\right|}{\frac{1}{2}\left|z-z_{j}\right|\left|z-\overline{z_{j}}\right|},
$$

where the $\operatorname{limit}_{z \rightarrow z_{j}}$ is meant in the circle $\partial \mathcal{U}$. The second result follows then from the fact that $\lim _{z \rightarrow \xi}\left|z^{2}-1\right| /|z-\bar{\xi}|$ is equal to 1 for every $\xi \in \partial \mathcal{U}$, except for $\xi=1$ and $\xi=-1$ for which it is equal to 2 . 
In view of the above, we are now able to relate the Lagrange polynomials associated with the sections $R_{k}$ and the set $G_{k}$, hence the Lebesgue functions associated with $R_{k}$ and $G_{k}$. First, we introduce the quotient notation

$$
q_{k}(z, \xi):=\frac{\left|w_{\overline{F_{k}}}(z)\right|}{\left|w_{\overline{F_{k}}}(\xi)\right|}, \quad z \in \partial \mathcal{U}, \quad \xi \in \partial \mathcal{U} \backslash F_{k} .
$$

Lemma 4.4 We have

$$
\mathbb{L}_{R_{k}} \leq 2 \mathbb{L}_{G_{k}} \sup _{\substack{z \in \partial \mathcal{U} \\ \xi \in G_{k}}} q_{k}(z, \xi)
$$

Proof: We denote by $l_{0}, \ldots, l_{k-1}$ the Lagrange polynomials associated with the section $R_{k}$ and by

$$
L_{0}, L_{1}, L_{(2,1)}, L_{(2,2)}, \cdots, L_{\left(2^{n}, 1\right)}, L_{\left(2^{n}, 2\right)}, L_{2^{n}+1}, \cdots, L_{k-1},
$$

the Lagrange polynomials associated with the set $G_{k}$ following the order given in 4.6). For convenience, we write the first polynomials as

$$
l_{j}(x):=\frac{W_{R_{k}}(x)}{W_{R_{k}}^{\prime}\left(r_{j}\right)\left(x-r_{j}\right)}, \quad x \in[-1,1],
$$

In view of Lemma 4.3 and identity 4.9 we have for $j=0, \ldots, k-1, z \in \partial \mathcal{U}$ and $x=\Re(z)$

$$
\left|l_{j}(x)\right|=\frac{1}{\alpha_{j}}\left|\frac{z^{2}-1}{\left(z-z_{j}\right)\left(z-\overline{z_{j}}\right)}\right| \frac{\left|w_{G_{k}}(z)\right|}{\left|w_{G_{k}}^{\prime}\left(z_{j}\right)\right|} \frac{\left|w_{\overline{F_{k}}}(z)\right|}{\left|w_{\overline{F_{k}}}\left(z_{j}\right)\right|}
$$

where $\alpha_{j}$ are defined as in Lemma 4.3. We observe that

$$
\left|\frac{z^{2}-1}{(z-\xi)(z-\bar{\xi})}\right|=\left|\frac{z-\bar{z}}{(z-\xi)(\bar{z}-\xi)}\right| \leq \frac{1}{|z-\xi|}+\frac{1}{|\bar{z}-\xi|}
$$

The last inequality applied with the real values $\xi=z_{0}=1$ and $\xi=z_{1}=-1$ and injected in 4.12 yields

$$
\left|l_{0}(x)\right| \leq q_{k}\left(z, z_{0}\right)\left|L_{0}(z)\right| \quad \text { and } \quad\left|l_{1}(x)\right| \leq q_{k}\left(z, z_{1}\right)\left|L_{1}(z)\right|
$$

Now for the indices $j=2, \ldots, 2^{n}$, since $z_{j}$ and $\overline{z_{j}}$ play symmetric roles in that $\Re\left(z_{j}\right)=$ $\Re\left(\overline{z_{j}}\right)=r_{j}$ and $z_{j}, \overline{z_{j}} \in G_{k}$, then one observes that 4.8 yields

$$
\left|w_{G_{k}}^{\prime}\left(z_{j}\right)\right|\left|w_{\overline{F_{k}}}\left(z_{j}\right)\right|=\frac{1}{2}\left|W_{R_{k}}^{\prime}\left(r_{j}\right)\right|=\left|w_{G_{k}}^{\prime}\left(\overline{z_{j}}\right)\right|\left|w_{\overline{F_{k}}}\left(\overline{z_{j}}\right)\right| .
$$

Taking this equality into account when injecting 4.13 into 4.12 and the fact that $\alpha_{j}=1$, we deduce

$$
\left|l_{j}(x)\right| \leq q_{k}\left(z, z_{j}\right) L_{(j, 1)}(z)+q_{k}\left(z, \overline{z_{j}}\right) L_{(j, 2)}(z)
$$


Finally for the indices $j=2^{n}+1, \ldots, k-1$, taking account of $|z-\bar{\xi}|=|\bar{z}-\xi|$ and the easily checked identity

$$
\left|w_{G_{k}}(z) w_{\overline{F_{k}}}(z)\right|=\left|w_{G_{k}}(\bar{z})\right|\left|w_{\overline{F_{k}}}(\bar{z})\right|,
$$

when injecting 4.13 into 4.12, we obtain

$$
\left|l_{j}(x)\right| \leq q_{k}\left(z, z_{j}\right) L_{j}(z)+q_{k}\left(\bar{z}, z_{j}\right) L_{j}(\bar{z}) .
$$

Summing the inequalities 4.14, 4.15) and 4.16), we conclude the proof.

In view of the previous lemma, we can derive Theorem 4.1 through a study of the growth of the quotients function $q_{k}$. By the structure of Leja sequences on $\mathcal{U}$, we have that $F_{k}=$ $E_{2^{n+1}, 2^{n}+k-1}$ is a $k^{\prime}$-Leja section with $k^{\prime}=k-\left(2^{n}+1\right)$ and $0<k^{\prime}<2^{n}$, therefore by (3.10), we derive

$$
q_{k}(z, \xi)=\frac{\left|w_{F_{k}}(\bar{z})\right|}{\left|w_{F_{k}}(\bar{\xi})\right|} \leq \frac{2^{n+1-p_{0}\left(k^{\prime}\right)}}{\bar{\xi}^{2 n}-e_{2^{n+1}}^{2^{n}}}
$$

Since $e_{2^{n+1}}$ is a $2^{n+1}$-root of -1 , then $e_{2^{n+1}}^{2^{n}}= \pm i$. As for $\xi \in G_{k}$, since $G_{k} \subset E_{2^{n+2}}=\mathcal{U}_{2^{n+2}}$ then $\xi^{2^{n}} \in\{1,-1, i,-i\}$. This shows that necessarily $\left|\bar{\xi}^{2^{n}}-e_{2^{n+1}}^{2^{n}}\right| \geq \sqrt{2}$, so that

$$
\sup _{\substack{z \in \mathcal{U} \\ \xi \in G_{k}}} q_{k}(z, \xi) \leq 2^{n+\frac{1}{2}-p_{0}\left(k^{\prime}\right)}
$$

This bound injected in 4.11 completes the proof of Theorem 4.1.

\section{Growth of the norms of the difference operators}

In this section, we focus our attention on the difference operators

$$
\Delta_{0}=I_{0}, \quad \text { and } \quad \Delta_{k}=I_{k}-I_{k-1}, \quad k \geq 1
$$

associated with interpolation on Leja sequences on $\partial \mathcal{U}$ and $\Re$-Leja sequences on $[-1,1]$. We are interested in estimating their norm

$$
\mathbb{D}_{k}:=\sup _{f \in C(X)-\{0\}} \frac{\left\|\Delta_{k} f\right\|_{L^{\infty}(X)}}{\|f\|_{L^{\infty}(X)}} .
$$

We write $\mathbb{D}_{k}(Z)$ when needed to emphasize the dependence on the sequence $Z$. It is immediate that $\mathbb{D}_{0}=\mathbb{L}_{0}=1$ and $\mathbb{D}_{k} \leq \mathbb{L}_{k}+\mathbb{L}_{k-1}$ any for $k \geq 1$. We shall sharpen the previous bound when $Z$ has a particular structure, for instance, if $Z$ is a Leja or an $\Re$-Leja sequence. Similar to the expression of Lebesgue constant in 2.12 , we can express $\mathbb{D}_{k}$ using Lagrange 
polynomials. Indeed, using Lagrange interpolation formula in $z_{0}, \ldots, z_{k}$, it can be easily checked that for any $k \geq 1$

$$
\Delta_{k} f(z)=\left(f\left(z_{k}\right)-\Pi_{Z_{k}} f\left(z_{k}\right)\right) \frac{w_{Z_{k}}(z)}{w_{Z_{k}}\left(z_{k}\right)}, \quad z \in X .
$$

This implies that

$$
\mathbb{D}_{k}=\sup _{z \in X} \frac{\left|w_{Z_{k}}(z)\right|}{\left|w_{Z_{k}}\left(z_{k}\right)\right|} \sup _{f \in C(X)-\{0\}} \frac{\left|f\left(z_{k}\right)-\Pi_{Z_{k}} f\left(z_{k}\right)\right|}{\|f\|_{L^{\infty}(X)}}
$$

The second supremum in the previous equality is obviously bounded by $1+\lambda_{Z_{k}}\left(z_{k}\right)$. This bound is actually attained: to see this, take $f$ a function in $C(X)$ having a maximum value equal to 1 , and satisfying $f\left(z_{k}\right)=-1$ and $f\left(z_{j}\right)=\frac{\left|l_{j}\left(z_{k}\right)\right|}{l_{j}\left(z_{k}\right)}$ for every $j=0, \ldots, k-1$ where $l_{0}, \ldots, l_{k-1}$ are the Lagrange polynomials associated with $E_{k}$. Therefore

$$
\mathbb{D}_{k}=\left(1+\lambda_{Z_{k}}\left(z_{k}\right)\right) \sup _{z \in X} \frac{\left|w_{Z_{k}}(z)\right|}{\left|w_{Z_{k}}\left(z_{k}\right)\right|} .
$$

The previous formula shows in particular that if $Z$ is a Leja sequence on $X$, then

$$
\mathbb{D}_{k}=1+\lambda_{Z_{k}}\left(z_{k}\right) .
$$

In particular, in view of the results on Leja sequences on the unit circle, more precisely (3.9), we have

Theorem 5.1 Let $E$ be a Leja section in $\partial \mathcal{U}$ with initial value $e_{0} \in \partial \mathcal{U}$. The norm of the difference operators associated with $E$ satisfy, $\mathbb{D}_{0}=1$ and for $k \geq 1$

$$
\mathbb{D}_{k} \leq 1+k
$$

Combining this result with (2.16), we obtain the following stability estimate for the multivariate interpolation operator.

Corollary 5.2 With $X=\mathcal{U}$ and $Z$ the Leja sequence with initial value $e_{0} \in \partial \mathcal{U}$, one has

$$
\mathbb{L}_{\Lambda} \leq(\#(\Lambda))^{2},
$$

for any lower set $\Lambda$.

The formula (5.5) is convenient in the case of Leja sequence since it yields exact values of the quantities $\mathbb{D}_{k}$. In the case of $\Re$-Leja sequences, we opt for a different expression of (5.5). From the formulas of Lagrange polynomials associated with $Z_{k}$, we may write (5.5) as

$$
\mathbb{D}_{k}=\left(\frac{1}{\left|w_{Z_{k}}\left(z_{k}\right)\right|}+\sum_{j=0}^{k-1} \frac{1}{\left|w_{Z_{k}}^{\prime}\left(z_{j}\right)\right|\left|z_{k}-z_{j}\right|}\right) \sup _{z \in X}\left|w_{Z_{k}}(z)\right| .
$$


We remark that $\left|w_{Z_{k}}\left(z_{k}\right)\right|=\left|w_{Z_{k+1}}^{\prime}\left(z_{k}\right)\right|$ and $\left|w_{Z_{k}}^{\prime}\left(z_{j}\right)\right|\left|z_{k}-z_{j}\right|=\left|w_{Z_{k+1}}^{\prime}\left(z_{j}\right)\right|$ for any $j=$ $0, \ldots, k-1$, we may then rewrite (5.5) in the more compact form

$$
\mathbb{D}_{k}=\left(\sum_{j=0}^{k} \frac{1}{\left|w_{Z_{k+1}}^{\prime}\left(z_{j}\right)\right|}\right) \sup _{z \in X}\left|w_{Z_{k}}(z)\right|
$$

Now, we let $R=\left(r_{j}\right)_{j \geq 0}$ be an $\Re$-Leja sequence. Using for this sequence the polynomials $W_{R_{k}}$ defined in (3.15) instead of $w_{R_{k}}$, we might rewrite 5.10 for $R$ as

$$
\mathbb{D}_{k}(R)=2 \beta_{k}(R) \sup _{x \in[-1,1]}\left|W_{R_{k}}(x)\right| \quad \text { where } \quad \beta_{k}(R):=\sum_{j=0}^{k} \frac{1}{\left|W_{R_{k+1}}^{\prime}\left(r_{j}\right)\right|}
$$

We propose to bound separately the quantities $\beta_{k}(R)$ and $\sup _{x \in[-1,1]}\left|W_{R_{k}}(x)\right|$ in this order.

Lemma 5.3 Let $R$ be a $\Re$-Leja sequence. We have $\beta_{2^{n}}(R)=\frac{1}{4}$ for any $n \geq 0$. For $k \geq 1$, such that $2^{n}<k<2^{n+1}$,

$$
\beta_{k}(R) \leq C \frac{2^{\sigma_{0}(k)}}{2^{p_{0}(k)}}, \quad C=\frac{1}{4} .
$$

where $\sigma_{0}(k)$ is the number of zeros in the binary expansion of $k$.

Proof: We first assume that $k=2 N \geq 4$ is an even integer. We have

$$
\begin{array}{r}
\beta_{k}(R)=\frac{1}{\left|W_{R_{2 N+1}}^{\prime}(1)\right|}+\frac{1}{\left|W_{R_{2 N+1}}^{\prime}(-1)\right|}+\frac{1}{\left|W_{R_{2 N+1}}^{\prime}(0)\right|}+ \\
\sum_{j=2}^{N}\left(\frac{1}{\left|W_{R_{2 N+1}}^{\prime}\left(r_{2 j-1}\right)\right|}+\frac{1}{\left|W_{R_{2 N+1}}^{\prime}\left(r_{2 j}\right)\right|}\right) .
\end{array}
$$

We introduce the shorthand $S=R^{2}$. Using Lemma 3.5, we deduce that

$$
\beta_{k}(R)=\frac{1}{\left|W_{S_{N+1}}^{\prime}(1)\right|}+\frac{1}{\left|W_{S_{N+1}}^{\prime}(-1)\right|}+\sum_{j=2}^{N} \frac{1}{\left|W_{S_{N+1}}^{\prime}\left(s_{j}\right)\right|}=\beta_{N}(S) .
$$

The same arguments implies that $\beta_{2}(R)=\beta_{1}(S)$, so that $\beta_{2 N}(R)=\beta_{N}(S)$ is valid for any $N \geq 1$. Since $S$ is also an $\Re$-Leja sequence, then the verification $\beta_{1}(S)=\frac{1}{4}$ for any $\Re$-Leja sequence $S$ implies the first result in the lemma $\beta_{2^{n}}(R)=\frac{1}{4}$ for any $n \geq 0$.

We now assume that $k=2 N+1 \geq 5$ is an odd integer. First, we isolate the last quotient in the the sum giving $\beta_{k}(R)$ and multiply the other quotients by $\frac{\left|r_{j}-r_{k+1}\right|}{\left|r_{j}-r_{k+1}\right|}$ yielding

$$
\beta_{k}(R)=\frac{1}{W_{R_{k}}\left(r_{k}\right)}+\sum_{j=0}^{k-1} \frac{\left|r_{j}-r_{k+1}\right|}{\left|W_{R_{k+2}}^{\prime}\left(r_{j}\right)\right|}
$$


Since $k=2 N+1=2(N+1)-1$ and $k+2=2(N+2)-1$, then regrouping the sum as in (5.13) and using Lemmas 3.5 and 3.6 , we deduce

$$
\begin{array}{r}
\beta_{k}(R)=\frac{2\left|r_{k}\right|}{\left|W_{S_{N+1}}\left(s_{N+1}\right)\right|}+\frac{\left|1-r_{2 N+2}\right|+\left|-1-r_{2 N+2}\right|}{2\left|W_{S_{N+2}}^{\prime}(1)\right|}+\frac{\left|r_{2 N+2}\right|}{\left|W_{S_{N+2}}^{\prime}(-1)\right|}+ \\
\left(\sum_{j=2}^{N} \frac{\left|r_{2 j-1}-r_{2 N+2}\right|+\left|r_{2 j}-r_{2 N+2}\right|}{2\left|W_{S_{N+2}}^{\prime}\left(s_{j}\right)\right|}\right)
\end{array}
$$

Since $|x-r|+|x+r| \leq 2$ for any $x, r \in[-1,1]$ and $r_{2 j-1}=-r_{2 j}$, for every $j \geq 2$, we deduce that

$$
\begin{array}{r}
\beta_{k}(R) \leq \frac{2}{\left|W_{S_{N+1}}\left(s_{N+1}\right)\right|}+\frac{1}{\left|W_{S_{N+2}}^{\prime}(1)\right|}+\frac{1}{\left|W_{S_{N+2}}^{\prime}(-1)\right|}+\sum_{j=2}^{N} \frac{1}{\left|W_{S_{N+2}}^{\prime}\left(s_{j}\right)\right|}= \\
\frac{1}{\left|W_{S_{N+1}}\left(s_{N+1}\right)\right|}+\beta_{N+1}(S) \leq 2 \beta_{N+1}(S)
\end{array}
$$

We introduce the sequence $\left(u_{k}\right)_{k \geq 1}$ defined by

$$
u_{k}:=\sup \left\{\beta_{k}(R): R \text { is an } \Re \text {-Leja sequence }\right\}, \quad k \geq 1
$$

Since $S=R^{2}$ is an $\Re$-Leja sequence, then in view of the previous discussion, we have $u_{1}=1 / 4$ and

$$
u_{2 N}=u_{N}, \quad u_{2 N+1} \leq 2 u_{N+1}, \quad N \geq 1
$$

The sequence $\left(u_{k}\right)_{k \geq 1}$ is bounded by the sequence with initial value $1 / 4$ that saturates the previous inequality. We introduce the sequence

$$
v_{k}=\frac{2^{\sigma_{0}(k)-p_{0}(k)}}{4}, \quad k \geq 1
$$

We have $p_{0}(2 N)=1+p_{0}(N)$ and $\sigma_{0}(2 N)=1+\sigma_{0}(N)$, hence $v_{2 N}=v_{N}$. Now given an even number $l \geq 1$, we have by binary subtraction,

$$
l=\underbrace{00 \ldots 0}_{p(l)} 1 \underbrace{\ldots 1}_{p(l)-1} \Longrightarrow 2 l-1=\underbrace{11 \ldots 1}
$$

where the root "... 1" to the right has not changed. Therefore the number of zeros in $l$ and $2 l-1$ are related by

$$
\sigma_{0}(l)-p_{0}(l)=\sigma_{0}(2 l-1)-1 .
$$

This applied with $l=2(N+1)$ for $N \geq 1$ implies $\sigma_{0}(N+1)-p_{0}(N+1)=\sigma_{0}(2 N+1)-1$, thus $v_{2 N+1}=2 v_{N+1}$. Since $v_{1}=\frac{1}{4}$, this shows that $\left(v_{k}\right)_{k \geq 1}$ is the saturation sequence that 
bounds $\left(u_{k}\right)_{k \geq 1}$ and completes then the proof.

In view of the above lemma, we are now able to provide a bound on the growth of the norms of the difference operators for $\Re$-Leja sequence.

Theorem 5.4 Let $R$ be a $\Re$-Leja sequence. For any $n \geq 0$ and for $k \geq 1$, such that $2^{n} \leq k<2^{n+1}$,

$$
\mathbb{D}_{k}(R) \leq 4^{n} \leq(k+1)^{2}
$$

Proof: We have by Lemma 4.7) that for $2^{n}+1<k<2^{n+1}+1$ and with $k^{\prime}=k-\left(2^{n}+1\right)$

$$
\left|W_{R_{k}}(x)\right|=\left|z^{2}-1\right|\left|w_{G_{k}}(z)\right|\left|w_{\overline{F_{k}}}(z)\right| \leq 2 \times 2^{\sigma_{1}\left(2^{n+1}+k^{\prime}\right)} 2^{\sigma_{1}\left(k^{\prime}\right)}=4 \times 4^{\sigma_{1}\left(k^{\prime}\right)},
$$

where we have used that $G_{k}$ and $F_{k}$ are respectively $\left\{2^{n+1}+k^{\prime}\right\}$-Leja and $k$-Leja section the unit circle and the second point in Theorem 3.2 . This result is also valid for the value $k=2^{n}+1$.

Since $0<k^{\prime}<2^{n}$, the number of ones in the binary expansion of $k^{\prime}$ satisfies $\sigma_{1}\left(k^{\prime}\right)=$ $\sigma_{1}\left(k^{\prime}+2^{n}\right)-1=\sigma_{1}(k-1)-1$. It can be checked using binary subtraction $\sigma_{1}(k-1)=\sigma_{1}(k)-1$ if $k$ is odd and $\sigma_{1}(k-1)=p_{0}(k)-1+\sigma_{1}(k)$ for $k$ even, therefore

$$
\sigma_{1}\left(k^{\prime}\right)+1=\sigma_{1}(k)+p_{0}(k)-1
$$

We deduce then from (5.11) and the previous lemma that

$$
\mathbb{D}_{k}(R) \leq 2 \times 4^{\sigma_{1}(k)+p_{0}(k)-1} \frac{2^{\sigma_{0}(k)-p_{0}(k)}}{4}=\frac{1}{4} 2^{\sigma_{1}(k)+p_{0}(k)} 2^{\sigma_{1}(k)+\sigma_{0}(k)} \leq \frac{1}{4}\left(2^{n+1}\right)^{2}=4^{n} .
$$

where we have used $\sigma_{1}(k)+p_{0}(k) \leq \sigma_{1}(k)+\sigma_{0}(k)=n+1$.

Combining this result with 2.16), we obtain the following stability estimate for the multivariate interpolation operator.

Corollary 5.5 With $X=[-1,1]$ and $Z$ an $\Re$-Leja sequence, one has

$$
\mathbb{L}_{\Lambda} \leq(\#(\Lambda))^{3},
$$

for any lower set $\Lambda$.

\section{References}

[1] L. Bialas-Ciez and J.P. Calvi, Pseudo Leja sequence, Ann. Mat. Pura Appl, (2012) 191, 53-75. 
[2] J.P. Calvi and V.M. Phung, On the Lebesgue constant of Leja sequences for the unit disk and its applications to multivariate interpolation, Journal of Approximation Theory 163-5, (2011), 608-622.

[3] J.P. Calvi And V.M. Phung, Lagrange interpolation at real projections of Leja sequences for the unit disk, Proceedings of the American Mathematical Society, 140(12): (2012), 4271-4284.

[4] A. ChKifa, On the Lebesgue constant of Leja sequences for the complex unit disk and of their real projection, Journal of Approximation Theory 166, (2013), 176-200.

[5] A. ChKifa, Méthodes polynomiales parcimonieuses en grande dimension. Application aux EDP Paramétriques, PhD thesis, Laboratoire Jacques Louis Lions.

[6] A. Chkifa, A. Cohen and C. Schwab, High-dimensional adaptive sparse polynomial interpolation and applications to parametric PDEs, Foundations of Computational Mathematic, (2013), 1-33

[7] A. Chkifa, A. Cohen and C. Schwab, Breaking the curse of dimensionality in parametric PDE's, to appear in J. Math Pures et Appliquées (2014).

[8] A. Chkifa, A. Cohen, R. DeVore and C. Schwab, Sparse adaptive Taylor approximation algorithms for parametric and stochastic elliptic PDEs, Mathematical Modelling and Numerical Analysis (2013), 47, 253-280.

[9] A. Cohen, R. DeVore and C. Schwab, Convergence rates of best $N$-term Galerkin approximations for a class of elliptic sPDEs, Foundations of Computational Mathematics 10 (2010), 615-646.

[10] A. Cohen, R. DeVore and C. Schwab, Analytic regularity and polynomial approximation of parametric and stochastic PDE's, Analysis and Applications 9 (2011), $11-47$.

[11] Ph.J. DAvis, Interpolation and Approximation, Blaisdell Publishing Company, (1963).

[12] V.K. Dzjadyk and V.V. Ivanov, On asymptotics and estimates for the uniform norms of the Lagrange interpolation polynomials corresponding to the Chebyshev nodal points, Analysis Mathematica, (1983) 9-11, 85-97, .

[13] R.A Devore and G.G Lorentz, Constructive approximation,(1993), Springer.

[14] J. Kuntzman, Méthodes numériques - Interpolation, dérivées, Dunod, Paris, 1959. 
[15] R. TAYLOR, Lagrange interpolation on Leja points, PhD thesis, University of South Florida, 2008.

Abdellah Chkifa

UPMC Univ Paris 06, UMR 7598, Laboratoire Jacques-Louis Lions, F-75005, Paris, France CNRS, UMR 7598, Laboratoire Jacques-Louis Lions, F-75005, Paris, France

chkifa@ann.jussieu.fr

Albert Cohen

UPMC Univ Paris 06, UMR 7598, Laboratoire Jacques-Louis Lions, F-75005, Paris, France CNRS, UMR 7598, Laboratoire Jacques-Louis Lions, F-75005, Paris, France cohen@ann.jussieu.fr 